American Journal of Pharmacology and Toxicology 3 (1): 100-110, 2008

ISSN 1557-4962

(C) 2008 Science Publications

\title{
Elucidating the Mechanism(s) of Hormesis at the Cellular Level: The Universal Cell Response
}

\author{
Paul S. Agutter \\ Theoretical and Cell Biology Consultancy, \\ 26 Castle Hill, Glossop, Derbyshire, SK13 7RR, UK
}

\begin{abstract}
Many environmental stressors elicit biphasic effects from single cells. Such cellular hormesis may be interpreted in terms of (a) the superimposition of simple biochemical processes or (b) the non-specific behaviour of the cell. The latter approach is emphasized in this article and identified with the universal cell response (UCR); however, the importance of identifying molecular-level concomitants of the UCR is also acknowledged. One difficulty is that when the dose of ligand is very low, mass-action assumptions become invalid and reliable analysis of receptor-ligand interactions requires knowledge of the binding mechanism; this difficulty is discussed. The UCR (cellular hormesis) and its possible underlying mechanisms are considered in the framework of a general scheme of cell life, which logically implies cellular homeostasis or homeorhesis. This framework may be particularly helpful for elucidating and perhaps quantifying conditioning hormesis (adaptation to stressors). The findings of studies on the UCR cannot be extrapolated unequivocally to hormesis in whole organisms or populations and cellular-level hormesis cannot be inferred from whole-organism hormesis. Nevertheless, it has been argued that a better understanding of the underlying cellular mechanisms, i.e., of the UCR, may facilitate the analysis of whole-organism and population data.
\end{abstract}

Key words: Universal cell response, cellular homeostasis, law of mass action

\section{INTRODUCTION}

Failure to establish a credible mechanistic explanation of hormesis may partly explain why the scientific community remained sceptical about the phenomenon for so long, though there were other contributing factors such as confusion with homeopathy ${ }^{[1-5]}$. However, the reality and generality of hormesis have now been established beyond reasonable doubt, thanks largely to (a) detailed evaluations of large bodies of information ${ }^{[6,7]}$ and (b) compelling evidence that standard statistical analyses of epidemiological, toxicological and pharmacological data on human and other populations can mislead (e.g. ${ }^{[8,9]}$ ). Moreover, mechanistic explanations have been suggested during the past few years (e.g. ${ }^{[2,3,10-12]}$ ). Nevertheless, the search for better understanding continues.

Hormesis is an operational term denoting a nonlinear dose-effect relationship: agents that cause a negative effect at high doses may have a positive effect at low ones and low doses may be protective or conditioning. However, the meanings of the term and its various near-synonyms have been obscured by inconsistent and confusing usage. A recent study ${ }^{[13]}$ suggested a simplified system of terminology to clarify the field and the recommendations of ${ }^{[13]}$ are followed here. Briefly: (1) X hormesis denotes the biphasic (beta- curve) effect of a physiological, chemical or physical (e.g. radiation) stressor, X. (2) Prior exposure to a low dose of $\mathrm{X}$ protects many systems against a larger dose of the same or similar stressor; this phenomenon is dubbed $X$ conditioning hormesis. Thus, $\mathrm{X}$ conditioning hormesis denotes an adaptive or protective effect by the stressor X. (3) The toxicity of a high stressor dose is sometimes ameliorated by subsequent exposure to a low dose of the same stressor. This type of phenomenon is dubbed $X$ postexposure conditioning hormesis ${ }^{[13]}$.

Such nomenclature is necessary because hormesis has been reported in various guises and under various names in almost every biological and biomedical discipline. The effects of a very wide range of physical and chemical stressors are related biphasically to dose and such relationships are qualitatively independent of the stressor agent, the endpoint measured and the system under investigation ${ }^{[14]}$. In much of the general discussion that follows, however, the $\mathrm{X}$ term will be omitted; the three (putatively related) types of phenomenon will be denoted simply by hormesis, conditioning hormesis and postexposure conditioning hormesis.

In the past, the field has (understandably) been dominated by toxicology and the resulting emphasis on biphasic dose-response relationships in populations may have diverted the attention of some researchers 
from physiological or cell-biological studies $^{[11]}$, yet similar relationships are observed at the cell level, e.g. in respect of proliferation, differentiation and transformation. How should they be interpreted? The increasing and decreasing parts of a biphasic curve quantifying the cellular response to a stressor may reflect a single underlying mechanism. Alternatively, hormesis may suggest an aggregate of many different intracellular processes that summate to increasing and decreasing effects at different stressor doses. The latter alternative has been lucidly discussed, for example, by Connolly and Lutz ${ }^{[15]}$. The former proposal - that general rather than specific mechanisms explain why cells of many different types show qualitatively similar effects (biphasic or $\beta$-curves ${ }^{[16]}$ ) when challenged with numerous different stimuli - is philosophically problematic: it seems contrary to the character of modern mainstream cell-biological research, which focuses on molecular details. This has led some authors to question the possibility of a general mechanism ${ }^{[15]}$. Others (e.g. ${ }^{[10,11]}$ ) hold that such a mechanism is probable, even inevitable for homeodynamic systems. Indeed, there seems to be a widespread expectation, or at least a hope, that a general mechanistic account of hormesis will also explain conditioning hormesis and perhaps also postexposure conditioning hormesis. Much of the present article will be devoted to this debate and its implications.

There is a related question: how - if at all - can biphasic dose-effect curves obtained from cell studies be related to those obtained from studies of multicellular organisms or of populations? Some brief remarks on this important issue will be added at the end of the article.

Analysis of low-dose response data entails mathematical and statistical difficulties at both the cell and population levels. These difficulties have been discussed in the literature (e.g. ${ }^{[8,9,17-19]}$ ) but they are not always considered when possible instances (or mechanisms) of hormesis are explored. This article therefore begins with a brief survey of the data interpretation problem.

\section{Receptor-ligand binding at low concentrations:}

Exogenous chemical agents are commonly presumed to interact with their biological target molecules in accordance with the law of mass action. However, when the dissociation constant is very low and the ligand concentration is at least one order of magnitude lower, the continuum assumption implicit in the law of mass action becomes invalid and a stochastic approach to receptor-ligand binding is require ${ }^{[17,18]}$. The coefficient of variation of ligand binding depends crucially on the reaction scheme assumed ${ }^{[18]}$ : in general, cooperative binding leads to greater variances than noncooperative binding. Moreover, the variance increases more or less exponentially with decreasing ligand concentration; this is important because a number of ligands have biological effects at femtomolar or attomolar levels ${ }^{[18,20,21]}$. These findings show that (a) statistical analyses of low-dose effects may be invalid if they are based on mass-action assumptions - indeed, linear extrapolation of dose-effect data to low concentrations is misguided in principle - and (b) the binding mechanism must be known before the data can be interpreted. Low-concentration binding kinetics do not specifically predict hormesis, but they show that mathematical analyses such as those presented in ${ }^{[18]}$ need to be taken into account if data from cell studies are to be interpreted soundly.

When populations of organisms (e.g. humans) are investigated, as in epidemiological and risk-assessment studies, these difficulties of data interpretation at the cellular level are compounded and straightforward statistical approaches may give rise to serious errors. The problems inherent in analyzing such data have been much discussed; two illustrative publications ${ }^{[8,9]}$ are mentioned here. Cedergreen et al. ${ }^{[8]}$ reviewed a number of statistical modelling approaches to population data and identified weaknesses in many of them. They proposed a new approach, the delta method (with freely available software) and demonstrated its applicability to crop growth and plant toxicological data, in several cases identifying hormetic effects that had not been apparent previously. May and Bigelow ${ }^{[9]}$ recommended the use of various statistical approaches, including ordinal reparameterization, fractional polynomials and splines, to any given body of population data in order to distinguish U- or J-shaped curves from linear relationships. They emphasized that the exposure variable is generally not uniformly distributed and individual differences in effect are likely.

The overall inference from such investigations is that low-dose data from both population studies and cell-biological experiments need to be interpreted with great caution, otherwise genuinely non-linear effects may be overlooked. The techniques described in the references cited above, notably ${ }^{[8,9,18]}$, are likely to be particularly useful, but they are not alone; in Stebbing's method for extracting growth-control system output, for example, the errors grow with each step so the raw data must be of high quality (errors $<5 \%)^{[19]}$. However, statistical issues will not be considered further in this article; it will be assumed that unequivocally biphasic 
effects to wide ranges of stimuli are commonplace among cells, organisms and populations.

\section{THE GENERAL AND THE SPECIFIC: HORMESIS AT THE CELLULAR LEVEL}

The focus in this section will be on cellular hormesis, the biphasic effects of stressors on naïve cells; i.e., it will be assumed that the cells have not been conditioned by pre-exposure to low (or high) doses. Conditioning hormesis will be discussed later.

Connolly and Lutz ${ }^{[15]}$ reasoned that any stressor agent is likely to have multiple effects on a cell and superimposition of these effects may yield a biphasic aggregate. According to these authors, many nonmonotonic dose-effect curves can potentially be explained in this way, so there is no basis for assuming that hormesis is underpinned by a single universal mechanism. One of their proposals, following the model proposed by Szabadi ${ }^{[22]}$, involved two receptors with different affinities for the same ligand and opposing effects on cell physiology (Fig. 1); this could indeed explain certain apparent instances of hormesis, and the authors cited illustrative examples from the literature. Another proposal involved gene transcription induced by a dimeric receptor with one endogenous and one xenobiotic ligand: homodimers were transcriptionally active but mixed-ligand receptor complexes were not, so the transcription rate responded biphasically to the dose of xenobiotic.

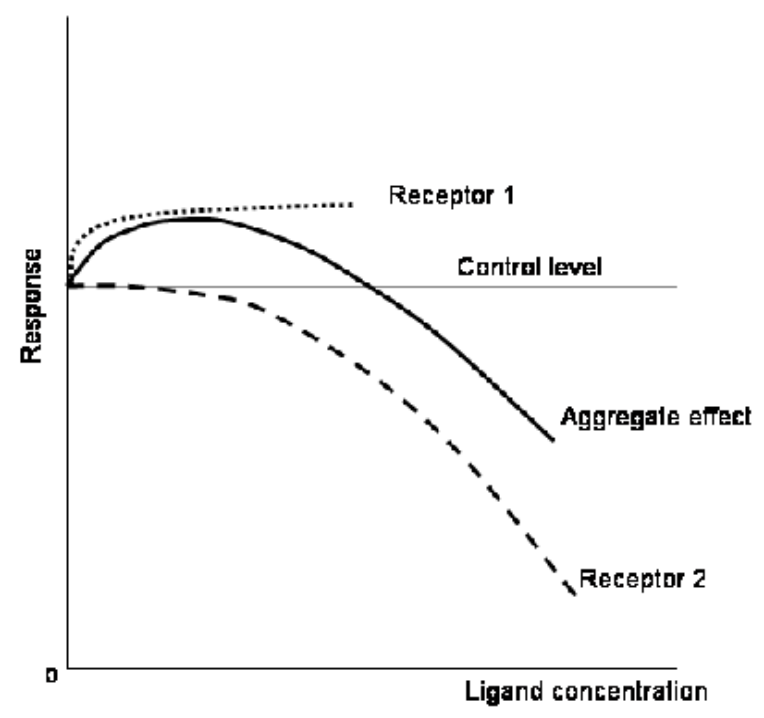

Fig. 1: Biphasic effect of a ligand with two different receptor types
Three comments must be made. First, the two aforementioned proposals are different in kind: one (potentially) entails an immediate effect of the ligand on cell behaviour, but the other entails a delayed effect because of the time required for de novo protein synthesis after initiation of transcription via receptor occupancy. This distinction will be further explored below. Second, the authors explicitly assumed that the law of mass action continues to apply as the ligand concentration tends towards zero, but this is not the case $^{[18]}$. The main argument of ${ }^{[15]}$ may not be seriously undermined by the fallacy, but issues such as the cooperativity of ligand binding need to be considered in relation to the examples cited. Third, although the search for molecular mechanisms underpinning any observed cell-biological event or process is considered an epistemological imperative, it behoves us not to focus so intensively on the minutiae of the explanens that we lose sight of the richness of the explicandum. Excessive attention to the parts might vitiate our understanding of the whole.

This caveat gains force when the general effects of external stimuli on cells are considered. In some cases, hormesis appears to result from wide-ranging intracellular changes rather than specific biochemical events; the influence of mild stress (e.g. heat stress) on ageing is an example ${ }^{[23-28]}$. Such stimuli, indeed a number of external stressors, have quite non-specific effects on protein glycation and oxidation and perhaps on the proteome as a whole $e^{[28,29]}$. To explicate such cases in terms of specific molecular processes would seem otiose. A different approach is needed.

More than half a century ago, Nasonov and his colleagues investigated the universal cell response (UCR). They showed ${ }^{[30]}$ that in cells of many different types from many different organisms, a wide variety of stressors (heat, mechanical stress, hydrostatic pressure, electric currents, general anaesthetics, altered $\mathrm{pH}$ and tonicity of the medium, heavy metal ions, hypoxia and sound irradiation) induce a standard array of changes in the turbidity, viscosity and biopotentials of cytoplasm and nucleoplasm, vital dye binding and resistance to the noxious stimulus. The UCR is biphasic. Low-intensity stimuli usually evoke decreases in turbidity, viscosity and vital dye binding, with concomitant increases in membrane potential and resistance to the harmful agent. The same stimuli at higher intensities have the opposite effects; also, the intracellular $\mathrm{pH}$ falls and electrolyte uptake increases. All these changes occur simultaneously at a given stimulus intensity, suggesting a single underlying mechanism, a final common pathway for the various intracellular processes initiated by the diverse stimuli. Mechanisms such as those 
proposed by Szabadi ${ }^{[22]}$ and by Connolly and Lutz ${ }^{[15]}$ may conceivably lead to, or participate in, such a final common pathway.

It has been suggested that hormesis at the cell level is a manifestation of, or is identical to, the $\mathrm{UCR}^{[31,32]}$. This is the position adopted in the present article.

\section{HORMESIS AND THE UNIVERSAL CELL RESPONSE}

Matveev $^{[33]}$ proposed that the hydrophobic phase volume of the cell initially decreases as the stimulus intensity rises from zero, then increases to far above the control or resting value as the stimulus intensity rises further. These changes in hydrophobic phase volume would account for the simultaneous changes in cell properties characteristic of the UCR. This proposal is corroborated by the fact that lipophilic xenobiotics such as valinomycin bind to a wide range of intracellular proteins $^{[33]}$ and that general anaesthetics with very different molecular structures act in more or less identical ways, binding to the hydrophobic domains of proteins and altering their structures ${ }^{[34,35]}$. Matveev's account of the UCR has been related to microrheological changes in the cytoplasm and nucleoplasm and to advances in understanding of the role of heat shock proteins in cellular effects of stress $^{[31]}$. There is indirect experimental support for a general cell-biological model of this kind ${ }^{[36,37]}$.

Another account, which on the face of it differs from that in ${ }^{[31,33]}$, has been proposed by Eïdus ${ }^{[31,37-39]}$. Eïdus initially studied $\mathrm{X}$-irradiation of mouse and Chinese hamster cells, including thymocytes, in vivo and in culture, measuring changes in (e.g.,) dry mass and loss of ability to form granules from neutral red dye ${ }^{[38,39]}$. These changes, which depended biphasically on the stressor dose, were convenient surrogates for the UCR, which he described as a defensive reaction identical to cellular hormesis. He subsequently extended his findings and his argument to chemical hormesis ${ }^{[40]}$. Essentially, Eïdus attributes the UCR (and ipso facto hormesis) to changes in compartmentalization resulting from alterations in the barrier functions of the plasma and intracellular membranes $^{[32]}$. These alterations change the local concentration gradients of some 250 different low molecular mass solutes, which inhibit a wide range of enzymes non-specifically; they also change the accessibility of the enzymes to these solutes. A low stressor dose changes the state of the plasma membrane lipid and allows inhibitory solutes to leak out of the cell, leading to increased enzyme activities. It also increases the intracellular $\mathrm{pH}$ by some 0.3-0.4 units, causing several genes to become transcriptionally active. Higher doses of the stressor destroy the intracellular solute gradients by impairing the barrier functions of the internal membranes, so there is widespread enzyme inhibition; the cell dies if the gradients are not restored to normal. Thus, low stressor doses increase various cell activities, including proliferation, but high doses have the opposite effect. Developments of Eïdus's view of hormesis and the UCR have included a novel interpretation of $\operatorname{apoptosis}^{[41]}$.

An interesting feature of this account is the emphasis on alkalinization of the cell, which is known to occur in association with many cellular functions including substratum adhesion and fertilization. Intracellular $\mathrm{pH}$ is also increased by stimulatory hormones, lectins and growth factors, and as mentioned earlier, high stressor doses have the opposite effect they acidify the cell. Few workers in the field seem to have considered the possible relationship between intracellular $\mathrm{pH}$ and hormesis.

\section{HORMESIS AND THE UCR VIEWED IN TERMS OF THE LIVING STATE OF THE CELL}

It is helpful to relate these proposals to the functional organization of the cell as a whole. An outline scheme of the living state at the cell level ${ }^{[42]}$ is reproduced in Fig. 2. Although this scheme does not in itself suggest new experimental approaches, it provides a conceptual framework within which different models of cellular hormesis, i.e. the UCR, can be interrelated.

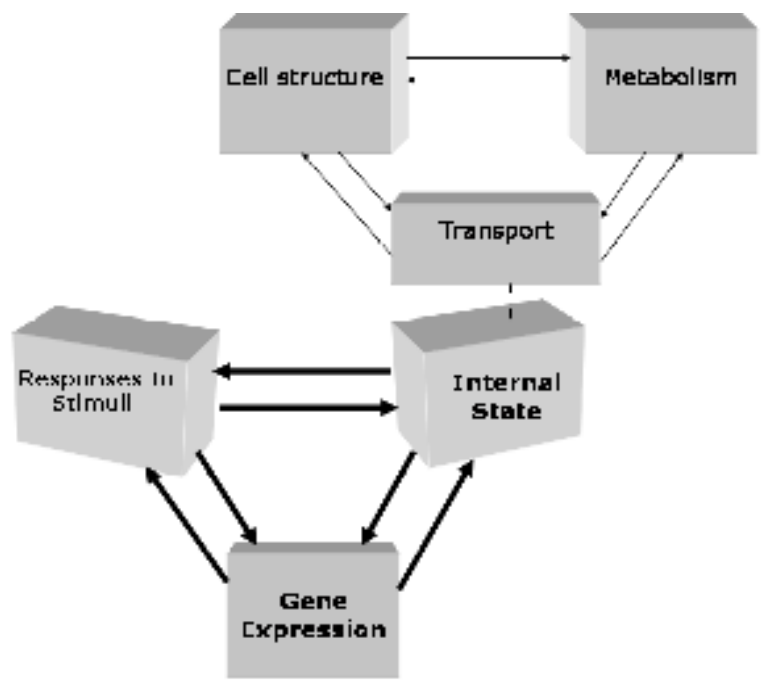

Fig. 2: Scheme of the living state of a cell 
According to this scheme, (a) the cell's internal state, (b) the responses to external stimuli and (c) the pattern of gene expression at any moment are reciprocally interdependent. Each of (a), (b) and (c) is determined by the other two. The internal state comprises all the cell's components and structures, all its metabolic activities and the totality of its transport processes (across and through membranes and through the interstices of the cytoplasm and organelles) at that moment. These three aspects of internal state are also reciprocally interdependent. Because changes in gene expression pattern or in the processing of extracellular stimuli or signals entail time-lags, this view of the cell implies that both homeostasis (the maintenance of a current state) and homeorhesis (progressive, programmed changes in state such as cell proliferation, differentiation or apoptosis) entail oscillations about the current state, or about the rate of change of state ${ }^{[42]}$. (Waddington's term homeorhesis was adopted in this context by Stebbing ${ }^{[43]}$; it denotes a stable pathway of change.) The time-lag involved in gene expression, mentioned during the earlier discussion of ${ }^{[15,22]}$, may be fundamental to our understanding of conditioning hormesis.

A low-intensity insult to the cell perturbs some aspect of the internal state. In all recent discussions of the UCR, this aspect is considered to be structure: the organization of the cytoskeleton and/or cytomatrix ${ }^{[31,33]}$ or the barrier functions of plasma and intracellular membranes ${ }^{[32]}$. Perturbations of both these kinds are likely to coincide, and the remainder of the internal state will also be affected almost simultaneously. Because the internal state is self-stabilizing by virtue of the reciprocal interdependence of its aspects or components $^{[42]}$, the perturbation will tend to be opposed. As Fig. 2 suggests, there will be consequent though less immediate changes in the pattern of gene expression and the processing of environmental signals.

\section{CONDITIONING HORMESIS AT THE CELL LEVEL}

These changes in gene expression and perhaps in the processing of signals, may constitute the basis for conditioning hormesis at the cell level (Fig. 3). Intuitively, conditioning hormesis may be pictured as a shift of the biphasic dose-effect curve to the right (cf. ${ }^{[19]}$ ), so that the effect becomes maximal at a higher stressor dose. Because of the difficulty of data interpretation at low doses, this simple representation of the phenomenon does not provide a reliable experimental method for quantifying the effect of conditioning (pre-exposure). A potentially more

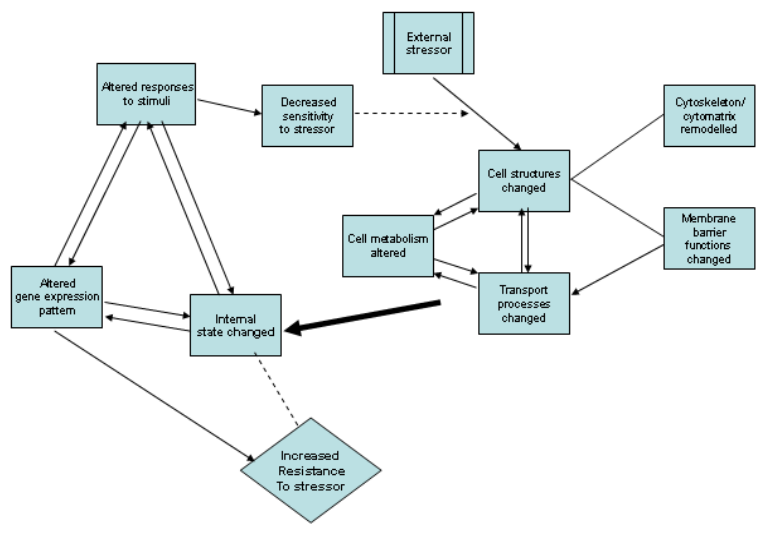

Fig. 3: A general scheme for conditioning hormesis

satisfactory experimental approach to quantification will be discussed in the next section.

The main function of the UCR (i.e. cellular hormesis) is considered to be evolutionary ${ }^{[2,32]}$ : it maintains the integrity of the genome in the face of environmental challenges, unless the challenge is too strong for the cell's defences. This view recalls Selye's account of the general adaptation syndrome in response to stress ${ }^{[44,45]}$, albeit at the cellular level. (A highintensity insult overwhelms the capacity of the internal state for self-correction and alters signal processing and the gene expression pattern, (a) directly and (b) indirectly via the transformed internal state. This results in the negative effects of high stressor doses.) The adaptive or defensive character of the UCR could depend primarily on (plasma) membrane barrier function $^{[32]}$ or on cytomechanics and heat-shock proteins ${ }^{[31]}$, or, of course, on both mechanisms, which are presumably concurrent and complementary (cf. Fig. 2).

Although it is helpful to explore conditioning hormesis in these general terms, the search for molecular mechanisms must remain paramount. One example of a molecular mechanism that could apply in some instances ${ }^{[15]}$ is the repair of DNA adducts formed by exogenous carcinogens. Doses of a carcinogen sufficient to induce endogenous repair processes, but too low to be significantly cytopathogenic, could thereby protect against subsequent higher doses. Moreover, because the repair processes are nonspecific, such low-dose exposure could diminish background DNA damage and therefore have a generally beneficial effect on the cell. Another proposal in ${ }^{[15]}$ could explain the biphasic effect of mutagens on the cell cycle (retardation at low doses and acceleration of high doses). Many other types of mechanism are also possible ${ }^{[15,46]}$ : Examples include the non-specific 
detoxifying effects of inducible cytochrome $\mathrm{P}_{450}$ isoforms, the general outcomes of heat shock protein production $^{[31]}$ and the effects of FOXO transcription factors on such characteristics as life-span ${ }^{[47]}$. An interesting challenge in the field is to relate these various mechanisms to the proposed primary events in the UCR after exposure to low doses of stressors and to the secondary changes in a cell's internal state that herald alterations in gene expression.

\section{ADAPTATION OF THE GROWTH CONTROL MECHANISM: GENERAL IMPLICATIONS FOR CONDITIONING HORMESIS}

A likely consequence of, or concomitant of, conditioning hormesis in proliferating cells is an increased mitotic rate, which could lead to altered (accelerated) growth of a multicellular organism. This is broadly consistent with the aforementioned general scheme of cell life ${ }^{[42]}$ (Fig. 2) and is fundamental to a well-established and widely-discussed explanation of hormesis $^{[10,11,19,43,48,49]}$. In these and other publications, Stebbing proposed that hormesis is a consequence of non-specific adaptive (homeostatic) effects. He did not mention the UCR, though it is interesting to recall that Nasonov and his School characterized the UCR as adaptive more than 50 years ago; they considered it to be an activation of defence mechanisms leading to overcompensation $^{[30]}$. Stebbing focused exclusively on the rate-sensitive mechanism of growth control and the capacity of this mechanism to adapt to sustained levels of an environmental stressor. Most of his experimental work was performed on marine coelenterates and yeasts.

Several other authors have also considered hormesis to be a consequence or manifestation of homeostasis (e.g. ${ }^{[50-54]}$ ), though homeorhesis has been less widely discussed. Both homeostasis and homeorhesis depend on non-specific correcting responses to perturbations involving feedback loops, which entail time-lags. Therefore, as outlined above, homeostasis involves oscillations of the state of the cell or organism about its norm, perhaps accompanied by a slight resetting of this norm after a perturbation. Similarly, homeorhesis involves oscillations and possible resetting in the wake of a perturbation: the normal or preferred rate of change of a variable such as the frequency of cell division may be altered.

Stebbing developed this argument in more detail and applied it to experimental data from growing or proliferating systems, as follows. The specific growth rate of an organism is measured as frequently as the precision of the data allows: if $\mathrm{N}$ is the initial number of organisms or cells examined, the specific growth rate is $(1 / \mathrm{N})(\mathrm{dN} / \mathrm{dt})$. It is measured at zero perturbation (no stressor load) to provide a control value and then at increasing stressor loads. Each value obtained is expressed as a fraction or percentage of the control value $^{[48,49]}$. When these normalized specific growth rates are plotted against time, the expected oscillations become apparent. At low stressor loads, the oscillations are gradually damped and are succeeded after a number of cell cycles by a sustained increase (continued growth). At high stressor loads, they yield to a sustained decrease (the cell or organism death rate exceeds the proliferation or growth rate). Crucially, the specific growth rate becomes less sensitive to a stressor agent if the system is pre-exposed to that agent for a sufficiently long period ${ }^{[49]}$ : the system adapts and resistance is increased. This approach therefore enables the experimenter to quantify conditioning hormesis in proliferating systems.

Of course, conditioning hormesis is also observed in circumstances under which cells are not proliferating, or their proliferation is not a relevant factor. However, Stebbing's analytical approach can presumably be generalized. For example, rates of differentiation of a cell type could be measured in control and stressorperturbed cultures or model organisms. Also, modern cell-biological techniques should make it possible to evaluate changes in UCR-related variables (e.g. cytoplasmic viscosity, $\mathrm{pH}$, turbidity), or in heat-shock protein production, membrane permeation rates, DNA adduct formation, etc. over short time-courses. If the chosen variable is denoted by $\mathrm{V}$ and its control (unperturbed) value by $\mathrm{V}^{*}$, a plot of $\left(1 / \mathrm{V}^{*}\right)(\mathrm{dV} / \mathrm{dt})$ against time would be expected to reveal oscillations of the kind reported by Stebbing ${ }^{[48,49]}$ and predicted in ${ }^{[42]}$. If plots of the mean value of $\left(1 / \mathrm{V}^{*}\right)(\mathrm{dV} / \mathrm{dt})$ against stressor dose were obtained from naïve cells and from cells pre-exposed to a low stressor loads, they could serve to quantify any instance of conditioning hormesis, as in $^{[48,49]}$ (Fig. 4). Such studies would help to elucidate the relationship between conditioning hormesis, the UCR and homeostasis/homeorhesis; in other words, confirm the generality of the adaptive response to stressors. In doing so, they may contribute to elucidating the mechanistic basis of the UCR (hormesis at the cellular level). 


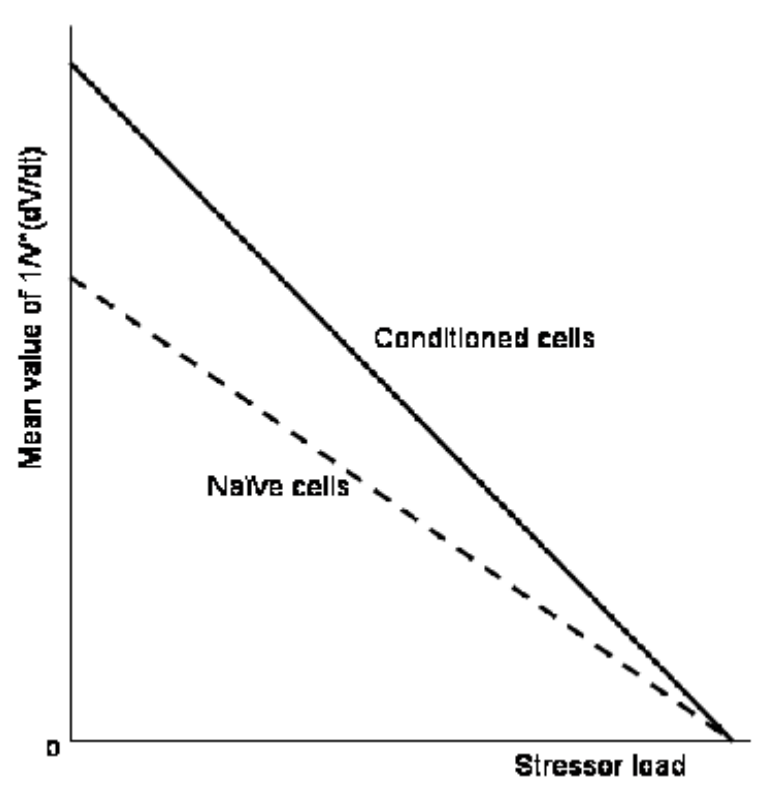

Fig. 4: A possible approach to quantifying an instance of conditioning hormesis

\section{POSTEXPOSURE CONDITIONING HORMESIS}

In the present state of knowledge, there is no obvious way of explicating cases in which the toxic effect of a stressor is ameliorated by subsequent exposure to a low dose of the same stressor. Postexposure conditioning hormesis does not appear to be accommodated by any of the accounts of cell-level processes surveyed in this article. Two questions need to be answered before further progress can be made on this matter. First, is postexposure conditioning hormesis, like hormesis and conditioning hormesis, observed in response to a very wide range of stressor types in numerous different biological systems, or is its occurrence more restricted? Unless the phenomenon is general, attempts to connect it mechanistically with the processes discussed in the foregoing sections may be futile. Second, is it observed at the cell level? To date, no publications seem to have addressed this second question. It may conceivably occur only at the wholebody level in multicellular organisms such as humans.

\section{RELATING HORMESIS AT THE WHOLE- ORGANISM AND POPULATION LEVELS TO HORMESIS AT THE CELL LEVEL (THE UCR)}

If there were consensus agreement about a mechanism or mechanisms explaining hormesis at the cell level, could this explanation be extrapolated to the whole-body level? Stebbing's rate-sensitive growth control mechanism has been identified in both unicellular organisms (marine yeasts ${ }^{[19]}$ ) and multicellular organisms (coelenterates ${ }^{[48]}$ ). More generally, such extrapolation might be justified if a drug or other stressor were known to elicit just one type of effect from a single target cell type, though even in such simple cases the reasoning could mislead. However, if the stressor could affect more than one type of target cell and evoke effects at different doses, extrapolation would be less straightforward. It has been shown that even closely-related cell types can differ in the effects of a stressor such as a carcinogen ${ }^{[55]}$. Moreover, the inverse argument would certainly be invalid: cell-level hormesis cannot be inferred from whole-organism hormesis. It could, for instance, be explained by opposite but linear effects of two different target cell types to different doses of the same agent, or, as the following example illustrates, by contradictory effects at the target-cell and whole-body levels.

Acute, non-fatal carbon monoxide (CO) poisoning causes lesions resembling deep venous thrombi and is reported to lead to pulmonary embolism ${ }^{[56,57]}$. The aetiology of deep venous thrombosis is now known to involve hypoxia-induced necrosis of the inner (parietalis) endothelium of the venous valve cusps, under conditions of alternating pulsatile and nonpulsatile venous blood flow; leukocyte infiltration of the necrotic cusp induces platelet recruitment and thrombus formation may be initiated ${ }^{[58]}$. Because $\mathrm{CO}$ in the circulation effectively causes hypoxaemia and therefore aggravates any tendency towards endothelial hypoxia, the likelihood of thromboembolism increases with increasing blood $\mathrm{CO}$ concentration until a fatal level is reached. However, linear extrapolation to very low $\mathrm{CO}$ doses would be seriously misleading. CO inhaled in minute doses, or produced endogenously by type I haem oxygenase, suppresses hypoxia-induced fibrinogenesis, probably by activating soluble guanylate cyclase in the vascular endothelial cells and thus preventing the hypoxic stimulation of plasminogen activator inhibitor- $1^{[59]}$. This has led to the suggestion that very-low-dose $\mathrm{CO}$ may have therapeutic value in cases of vascular ischaemia or hypoxaemia and, inter alia, in preventing thromboembolism ${ }^{[60]}$.

Thus, the relationship between non-fatal CO toxicity and venous thromboembolism would appear to be a classic though unacknowledged example of hormesis, but it does not admit of extrapolation to the cellular level. The alleged protective low-dose effect certainly involves a molecular effect in a specific target cell type (vascular endothelial cells), but the toxic higher-dose effect involves whole-body changes in 
blood oxygenation, increasing the likelihood of hypoxaemia in the venous valve pockets ${ }^{[58]}$. This counter-example illustrates the danger of assuming that all instances of whole-body hormesis can be explained at the cellular level.

For many toxins such as carcinogens, threshold doses can be identified for individual organisms; the dose-effect relationships are markedly sigmoidal. The absence of (carcinogenic or other) effect by subthreshold doses is not readily explained, though epigenetic rather than genotoxic mechanisms have been proposed $^{[60]}$ and it may be possible to relate these to the UCR. However, the pitfalls inherent in inferring celllevel effects from whole-body data indicate that such proposals require careful scrutiny, as the foregoing discussion of carbon monoxide and venous thrombosis illustrates.

Inter-individual differences in genetic predisposition and lifestyle or environment confer differences in threshold dose for any stressor ${ }^{[9,61]}$. Therefore, irrespective of the mode of action of the stressor, no threshold dose can be defined for an experimental group or sampled population. Differences in individual susceptibility to toxins determine the dose-effect relationship and this has implications for decisions about exposure limits ${ }^{[62]}$. The problem is exacerbated because, in reality, human and other populations are exposed to combinations of environmental stressors that may act synergistically or antagonistically. Nevertheless, detailed investigations of this practical issue have shown that an understanding of the mechanism of action of each stressor agent involved is of great value for data analysis ${ }^{[63]}$. This implies that the elucidation of hormesis at the cellular or single-cell organism level (i.e. the UCR) is likely to yield practical benefits in toxicological, epidemiological and environmental studies, reservations about the extrapolation of cell-biological data notwithstanding.

\section{CONCLUSIONS}

Although the effects of low doses of stressors on cells require careful interpretation, taking account for example of the mechanism of receptor-ligand binding, biphasic dose-effect relationships to a wide range of stressors are common to all cell types examined. In some cases at least, these relationships may be explained by the superimposition of simple biochemical processes that are presumed, individually, to be linearly related to dose. More generally, however, they seem to require explanation in terms of the non-specific Universal Cell Response (UCR). The various simple biochemical processes may be considered to lead to a final common pathway that is integral to the UCR.

Recently, two mechanistic accounts of the UCR have been proposed and have been explicitly related to hormesis. These two accounts appear complementary when viewed in terms of a general scheme of cell life and this perspective leads naturally to an understanding of conditioning hormesis. One well-studied example of conditioning hormesis, the adaptation of growth control mechanisms to a stressor, draws explicit attention to the general character of cellular homeostasis and homeorhesis. It leads to a method for quantifying conditioning hormesis that may be generalizable and could prove valuable in further investigations of the UCR.

Hormesis at the cell level is therefore a well established phenomenon and current research seems likely to elucidate the mechanism(s) involved. Such research will not directly explicate hormesis at the whole-organism or population levels, but knowledge of mechanisms may nevertheless be valuable for the analysis and interpretation of individual and population data.

\section{ACKNOWLEDGMENTS}

The author thanks Professor E.J. Calabrese, Professor V.V. Matveev, Dr. A.R.D. Stebbing and Professor D.N. Wheatley for helpful and encouraging comments on drafts of this article.

\section{REFERENCES}

1. Morré, D.J., 2000. Chemical hormesis in cell growth: A molecular target at the cell surface. J. Appl. Toxicol., 20: 157-163.

2. Forbes, V.E., 2000. Is hormesis an evolutionary expectation? Fuct. Ecol., 14: 12-24.

3. Rozman, K.K. and J. Doull, 2003. Scientific foundations of hormesis. Part 2. Maturation, strengths, limitations and possible applications in toxicology, pharmacology and epidemiology. Crit. Rev. Toxicol., 33: 451-462.

4. Calabrese, E.J., 2005. Paradigm lost, paradigm found: The re-emergence of hormesis as a fundamental dose response model in the toxicological sciences. Environ. Pollut., 138: 379-411.

5. Calabrese, E.J., J.W. Staudenmeyer and E.J. Stanek, 2006. Drug development and hormesis: Changing conceptual understanding of the dose response creates new challenges and opportunities for more effective drugs. Curr. Opin. Drug. Discovery Dev., 9: 117-123. 
6. Calabrese, E.J. and L.A. Baldwin, 2001. The frequency of U-shaped dose responses in the toxicological literature. Toxicol. Sci., 62: 330-338.

7. Calabrese, E.J., J.W. Staudenmeyer, E.J. Stanek III and G.R. Hoffmann, 2006. Hormesis outperforms threshold model in National Cancer Institute antitumor drug screening database. Toxicol. Sci., 94: 368-378.

8. Cedergreen, N., C. Ritz and J.C. Streibig, 2005. Improved empirical models describing hormesis. Envir. Toxicol. Chem., 24: 3166-3172.

9. May, S. and C. Bigelow, 2005. Modeling nonlinear dose-response relationships in epidemiologic studies: statistical approaches and practical challenges. Dose-Response, 3: 474-490.

10. Stebbing, A.R.D., 2000. Hormesis: interpreting the beta curve using control theory. J. Appl. Toxicol., 20: 93-101.

11. Stebbing, A.R.D., 2003. A mechanism for hormesis - a problem in the wrong discipline. Crit. Rev. Toxicol., 33: 463-467.

12. Kitchin, K.T., 2002. Defining, explaining and understanding hormesis. Hum. Exp. Toxicol., 21: 91-97.

13. Calabrese, E.J., K.A. Bachmann, A.J. Bailer, P.M. Bolger, J. Borak, L. Cai, N. Cedergreen, M.G. Cherian, C.C. Chiueh, T.W. Clarkson, P.R. Cook, D.M. Diamond, D.J. Doolittle, M.A. Dorato, S.O. Duke, L. Feinendegen, D.E. Gardner, R.W. Hart, K.L. Hastings, A.W. Hayes, G.R. Hoffmann, J.A. Ives, Z. Jaworowski, T.E. Johnson, W.B. Jonas, N.E. Kaminski, J.G. Keller, J.E. Klaunig, T.B. Knudsen, W.J. Kozumbo, T. Lettieri, S.Z. Liu, A. Maisseu, K.I. Maynard, E.J. Masoro, R.O. McClellan, H.M. Mehendale, C. Mothersill, D.B. Newlin, H.N. Nigg, F.W. Oehme, R.F. Phalen, M.A. Philbert, S.I. Rattan, J.E. Riviere, J. Rodricks, R.M. Sapolsky, B.R. Scott, C. Seymour, D.A. Sinclair, J. SmithSonneborn, E.T. Snow, L. Spear, D.E. Stevenson, Y. Thomas, M. Tubiana, G.M. Williams and M.P. Mattson, 2007. Biological stress response terminology: Integrating the concepts of adaptive response and preconditioning stress within a hormetic dose-response framework. Toxicol. Appl. Pharmacol., 222: 122-128.

14. Calabrese, E.J. and L.S. Baldwin, 1997. Chemical Hormesis: Scientific Foundations, Documentation and Implications for Risk Assessment. School of Public Health, Environmental Health Sciences, University of Massachusetts, Amherst, MA.
15. Connolly, R.B. and W.K. Lutz, 2004. Nonmonotonic dose-response relationships: Mechanistic basis, kinetic modeling and implications for risk assessment. Toxicol. Sci., 77: 151-157.

16. Townsend, J.F. and T.D. Luckey, 1960. Hormoligosis in pharmacology. JAMA., 173: 44-48.

17. Gurevich, K.G., 2001. Low doses of biologically active substances: effects, possible mechanisms and features. Cell Biol. Internat., 25: 475-484.

18. Gurevich, K.G., P.S. Agutter and D.N. Wheatley, 2003. Stochastic description of the ligand-receptor interaction of biologically active substances as extremely low doses. Cell Signalling, 15: 447-453.

19. Stebbing, A.R.D., J.P. Norton and M.D. Brinsley, 1984. Dynamics of growth control in a marine yeast subjected to perturbation. J. Gen. Microbiol., 130: $1799-1808$.

20. Leinders-Zufall, T., A.P. Lane, A.C. Puche, W. Ma, M.V. Novotny, M.T. Shipley and F. Zufall, 2000. Ultrasensitive pheromone detection by mammalian vomeronasal neurons. Nature, 405: 792-796.

21. Williamson, S.A., R.A. Knight, S.L. Lightman and J.R. Hobbs, 1987. Differential effects of betaendorphin fragments on human natural killing. Brain Behav. Immunol., 1: 329-335.

22. Szabadi, E., 1977. Model of 2 functionally antagonistic receptor populations activated by same agonist. J. Theor. Biol., 69: 101-112.

23. Norgaard. R., M. Kassem and S.I.S. Rattan, 2006. Heat shock-induced enhancement of osteoblastic differentiation of hTERT-immortalized mesenchymal stem cells. Ann. N.Y. Acad. Sci., 1067: 443-447.

24. Rattan, S.I.S., 2005. Hormetic modulation of aging and longevity by mild heat stress. Dose Response, 3: 533-546.

25. Rattan, S.I.S. and B.F.C. Clark, 2005. Understanding and modulating aging. Life, 57: 297-304.

26. Kyriazis, M., 2005. Clinical anti-aging hormetic strategies. Rejuvenat. Res., 8: 96-100.

27. Fonager, J., R. Beedholm, B.F.C. Clark and S.I.S. Rattan, 2002. Mild stress-induced stimulation of heat-shock protein synthesis and improved functional ability of human fibroblasts undergoing aging in vitro. Exp. Gerontol., 37: 1223-1228.

28. Verbeke, P., B.F.C. Clark and S.I.S. Rattan, 2000. Modulating cellular aging in vitro: hormetic effects of repeated mild heat stress on protein oxidation and glycation. Exp. Gerontol., 35:.787-794. 
29. Randic, M. and E. Estrada, 2005. Order from chaos: Observing hormesis at the proteome level. J. Proteome Res., 4: 2133-2136.

30. Nasonov, D.N., 1962. Local Reaction of Protoplasm and Gradual Excitation (English Translation by Halpern, Y.S.). National Science Foundation, Washington DC. Available from the Office of Technical Services, US Department of Commerce.

31. Agutter, P.S., 2007. Cell mechanics and stress: From molecular details to the universal cell response and hormesis. BioEssays, 29: 324-333.

32. Eïdus, L.Kh., 2005. On the mechanism of the nonspecific cell response to the action of damaging agents and the nature of hormesis. Biofizika, 50: 693-703.

33. Matveev, V.V., 2005. Protoreaction of protoplasm. Cell. Mol. Biol., 51: 715-723.

34. Franks, N.P. and E. Honore, 2004. The TREK K2P channels and their role in general anaesthesia and neuroprotection. Trends Pharmacol. Sci., 35: 601-608.

35. Franks, N.P., 2006. Molecular targets underlying general anaesthesia. Br. J. Pharmacol., 147: S72-81.

36. Arumugam, T.V., M. Gleichmann, S.C. Tang and M.P. Mattson, 2006. Hormesis/preconditioning mechanisms, the nervous system and aging. Ageing Res. Rev., 5: 165-178.

37. Rohrbach, S., B. Niemann, A.M. Abushouk and J. Holtz, 2006. Caloric restriction and mitochondrial function in the ageing myocardium. Exp. Gerontol., 41: 525-531.

38. Eïdus, L.Kh., 1994. The mechanism of the initiation of low-dose effects. Radiats. Biol. Radioecol., 34: 748-758.

39. Eïdus, L.Kh., 1996. A single mechanism for the initiation of different effects of low doses of ionizing radiation. Radiat. Biol. Radioecol., 36: 874-882.

40. Eïdus, L.Kh. and V.L. Eïdus, 2001. Problems of the mechanism of radiation and chemical hormesis. Radiats. Biol. Radioecol., 41: 627-630.

41. Eïdus, L.Kh., 1997. Is apoptosis a programmed cell death? Radiats. Biol. Radioecol., 37: 527-532.

42. Agutter, P.S. and D.N. Wheatley, 2007. About Life: Concepts in Modern Biology. Springer Dordrecht, pp: 91-103.

43. Stebbing, A.R.D., 1998. A theory for growth hormesis. Mutat. Res., 403: 249-258.

44. Selye, H, 1946. The general adaptation syndrome and the diseases of adaptation. J. Clin. Endocrinol., 6: 117-231.
45. Goldstein, D.S. and I.J. Kopin, 2007. Evolution of concepts of stress. Stress, 10: 109-120.

46. Arumugam, T.V., M. Gleichmann, S.C. Tang and Mattson, 2006. Hormesis/Preconditioning mechanisms, the nervous system and aging. Ageing Res. Rev., 5: 165-178.

47. Daitoku, H. and A. Fukamizu, 2007. FOXO transcription factors in the regulatory networks of longevity. J. Biochem. (Tokyo), 141: 769-774.

48. Stebbing, A.R.D., 1987. Growth hormesis: A byproduct of control. Health Phys., 52: 543-547.

49. Stebbing, A.R.D., 2003. Adaptive responses account for the $\beta$-curve - hormesis is linked to acquired tolerance. Nonlinearity Biol. Tox. Med., 1: 493-511.

50. Calabrese, E.J. and L.A. Baldwin, 2001. Hormesis: A generalizable and unifying hypothesis. Crit. Rev. Toxicol., 31: 353-424.

51. Calabrese, E.J., 2001. Overcompensation stimulation: A mechanism for hormetic effects. Crit. Rev. Toxicol., 31: 425-470.

52. Fabrikant, J.I., 1987. Adaptation of cell renewal systems under continuous irradiation. Health Phys., 52: 561-570.

53. Cox, L.A., 2005. A model of cytotoxic doseresponse nonlinearities arising from adaptive cell inventory management in tissues. Dose-Response, 3: 491-507.

54. Van der Woude, H., G.M. Alink and I.M.C.M. Rietjens, 2005. The definition of hormesis and its implications for in vitro to in vivo extrapolation and risk assessment. Crit. Rev. Toxicol., 35: 603-607.

55. Lutz, W.K., S. Vamvakas, A. Kopp-Schneider, J. Schlatter and H. Stopper, 2002. Deviation from additivity in mixture toxicity: relevance of nonlinear dose-response relationships and cell line differences in genotoxicity assays with combinations of chemical mutagens and gammaradiation. Environ. Health Perspect., 110: 915-918.

56. Philip K. Drinker, 1938. Carbon monoxide asphyxia. Oxford University Press, New York, pp: $124 \mathrm{ff}$.

57. Wilhelm F. van Ottingen, 1941. Studies on the mechanism of carbon monoxide poisoning as observed in dogs anesthetized with sodium amytal. United States Public Health Service, Washington DC.

58. Malone, P.C. and P.S. Agutter, 2006. The aetiology of deep venous thrombosis. Quart. J. Med., 99: 581-593. 
59. Fujita, T., K. Toda, A. Karimova, S.F. Yan, U. Naka, S.F. Yet and D.J. Pinsky, 2001. Paradoxical rescue from ischemic lung injury by inhaled carbon monoxide driven by derepression of fibrinolysis. Nature Med., 7: 597-604.

60. Mishra, S., T. Fujita, V.N. Lama, D. Nam, H. Liao, M. Okada, K. Minamoto, Y. Yoshikawa, H. Harada, H. and D.J. Pinsky, 2006. Carbon monoxide rescues ischemic lungs by interrupting MAPK-driven expression of early growth response 1 gene and its downstream target genes. Proc. Natl. Acad. Sci. USA, 103: 5191-5196.

61. Lutz, W.K. 2000. A true threshold dose in chemical carcinogenesis cannot be defined for a population, irrespective of the mode of action. Hum. Exp. Toxicol., 19: 566-568.
62. Lutz, W.K., 2002. Differences in individual susceptibility to toxic effects of chemicals determine the dose-response relationship and consequences of setting exposure standards. Toxicol. Lett., 126: 155-158.

63. Lutz, W.K., O. Tiedge, R.W. Lutz and H. Stopper, 2005. Different types of combination effects for the induction of micronuclei in mouse lymphoma cells by binary mixtures of the genotoxic agents MMS, MNU and genistein. Toxicol. Sci., 86: 318-323. 